\title{
MODEL DEVELOPMENT OF SUPERSONIC TROUGH WIND WITH SHOCKS
}

\section{J. M. GREBOWSKY}

(NASA-TM-X-65848) MODEL DEVELOPMENT OF SUPERSONIC TROUGH UIND WITH SHOCKS J.M. Grebowsky (NASA) Feb. 197231 p CSCL 20D 


\section{MODEL DEVELOPMENT OF SUPERSONIC TROUGH WIND WITH SHOCKS}

J. M. Grebowsky

February 1971

Laboratory for Planetary Atmospheres

Goddard Space Flight Center

Greenbelt, Maryland 


\section{PRECEDING PAGE BLANE NOT FLLMEL}

CONTENTS

Page

ABSTRACT $\ldots \ldots \ldots \ldots \ldots \ldots \ldots \ldots \ldots \ldots \ldots \ldots \ldots \ldots \ldots \ldots \ldots$

Introduction $\ldots \ldots \ldots \ldots \ldots \ldots \ldots \ldots \ldots \ldots \ldots \ldots \ldots \ldots \ldots$

Theory ............................ 3

Fixed L Coordinate $\ldots \ldots \ldots \ldots \ldots \ldots \ldots \ldots \ldots \ldots \ldots \ldots$

Cross L Drift $\ldots \ldots \ldots \ldots \ldots \ldots \ldots \ldots \ldots \ldots \ldots \ldots \ldots \ldots$

Field Line Contraction . . . . . . . . . . . . . . . . . 12

Expanding Flux Tube $\ldots \ldots \ldots \ldots \ldots \ldots \ldots \ldots \ldots \ldots \ldots \ldots \ldots$

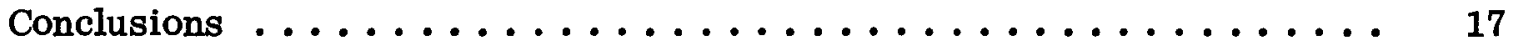

Bibliography $\ldots \ldots \ldots \ldots \ldots \ldots \ldots \ldots \ldots \ldots \ldots \ldots \ldots \ldots \ldots$ 
PRECEDING PAGE BLANK NOT FILMED

\title{
MODEL DEVELOPMENT OF SUPERSONIC TROUGH \\ WIND WITH SHOCKS
}

J. M. Grebowsky

\begin{abstract}
The time dependent one dimensional hydrodynamic equations describe the evolution of the thermal plasma flow along closed magnetic field lines outside of the plasmasphere. The convection of the supersonic polar wind onto a closed field line results in the assumed formation of collisionless plasma shocks. These shocks move earthward as the field line with its "frozen-in" plasma remains fixed or contracts with time to smaller $\mathrm{L}$ coordinates. The high equatorial plasma temperature (of the order of electron volts) produced by the shock process decreases with time if the flow is isothermal but it will increase if the contraction is under adiabatic conditions. Assuming adiabaticity a peak in the temperature forms at the equator in conjunction with a depression in the ion density. After an initial contraction, if the flux tube drifts to higher $L$ coordinates the direction of the shock motion can be reversed so that the supersonic region will expand along the field line towards the state characterizing the supersonic polar wind. A rapid expansion will lower the equatorial density while the temperature decreases with time under adiabatic but not isothermal conditions.
\end{abstract}




\section{MODEL DEVELOPMENT OF SUPERSONIC TROUGH}

\section{WIND WITH SHOCKS}

\section{$\underline{\text { Introduction }}$}

It is well established that the plasmasphere abruptly terminates at a magnetic field aligned shell which intersects the equatorial plane at a geocentric distance of a few earth radii. This boundary, the plasmapause, corresponding to a rapid decrease of the light ion density with increasing $\mathrm{L}$ coordinate also appears to separate two regions of distinctly different light ion flow behavior (Mayr et al., 1970; Brinton et al., 1971, Banks et al., 1971). Within the plasmasphere the macroscopic flow of thermal $\mathrm{H}^{+}$ions along the field lines is subsonic with the direction of the flow either away from or toward the earth depending on the phase of the diurnal rotation. On the other hand, the closed field lines outside of the plasmasphere (i.e., the field lines associated with the light ion trough) are characterized by regions of upward directed flows of the thermal $\mathrm{H}^{+}$ions which approach or even exceed the sound speed.

The upward directed supersonic $\mathrm{H}^{+}$fluxes along the trough field lines are analogous to and are directly related to the supersonic polar wind flowing along open polar lines (Banks and Holzer, 1969a). The relation between the trough wind and the polar wind is depicted in Figure 1. Through field line reconnection the supersonically flowing polar wind convects onto closed magnetic field lines extending to the earth's magnetospheric tail. The upward directed supersonic $\mathrm{H}^{+}$fluxes from the northern 
and southern hemispheres will initially merge near the magnetic equator. Instabilities will prevent the interpretation of these oppositely directed streams (Koons and Schulz, 1970; Schulz and Koons, 1972) converting the ordered flow motion into thermal energy.

If the transition from supersonic to subsonic flow along a trough field line occurs rapidly enough to be treated as a shock discontinuity (Banks et al., 1971) two collisionless shocks - one in each hemisphere - will be formed initially on the closed field line resulting from reconnection in the magnetosphere tail. These shocks will propagate away from the equator along the field line towards the earth as the ambient plasma, frozen to the magnetic field lines, drifts towards the dayside magnetopause where the closed field line opens via connection to the interplanetary field resulting in the expansive polar wind.

Banks et al. (1971) have computed the motion of these shocks along a magnetic dipole field line which is fixed in space - i.e., along a field line characterized by a constant $\mathrm{L}$ coordinate. These computations yielded the important result that for an isothermal supersonic $\mathrm{H}^{+}$flow along a fixed $\mathrm{L}$ field line the characteristic travel time of the shocks from the equator to the base of the protonosphere can be much larger than the average time required for a closed flux tube with its associated plasma to convect from the magnetosphere tail to the dayside magnetopause. Hence within the light ion trough the lower protosphere should on the average be characterized by an upward directed supersonic flow of the $\mathrm{H}^{+}$ions. 
This paper will expand upon the study of Banks et al. (1971) by explicitly evaluating the protonospheric flow development along a closed trough field line for both isothermal and adiabatic flow states. Also since the ambient plasma can drift perpendicular to the direction of the magnetic field, the compression or expansion of a magnetic flux tube and its associated plasma as occurs during a convection cycle must be considered in a complete study of the trough flow. The effects of the cross $\mathrm{L}$ drift on the supersonic flow of $\mathrm{H}^{+}$and on the shock motion will be explored.

\section{Theory}

The hydrodynamic description has been shown by Holzer et al. (1971) to be a good approximation to the supersonic flow state characterizing the polar wind. Such a description was used in previous studies of the polar wind (Banks and Holzer, 1969a, 1969b; Banks et al., 1971) and will be used to approximate the supersonic flow region on trough field lines. The subsonic flow downstream of the shocks has been randomized by the shock process and will be characterized by an isotropic pressure so that the flow everywhere along the trough field line is describable by the hydrodynamic relations.

Previous model studies of the supersonic flow of the polar and trough winds used the steady state one dimensional form of the hydrodynamic equations to describe the flow along a field line. This is valid when the $\mathrm{L}$ coordinate is constant and when steady state conditions prevail at the base of the field line because any departure of the supersonic region from static equilibrium results 
in a rapid relaxation to the steady state configuration. However, fixing the frame of reference in the plane containing the trough field line under investigation, the field aligned subsonic flow associated with the shock transition requires the use of the time dependent hydrodynamic equations since the shock position varies with time. Hence attention will be devoted to solving this form of the flow equations. The one dimensional time dependent equations can also be extended to study the flow variations produced by cross $L$ drifts - this is to be considered in a later section.

Along a field line (assumed dipolar) characterized by an $\mathrm{L}$ coordinate fixed in time, the hydrodynamic equations can be resolved into components along the field direction with two independent variables: the time $t$ and the distance $s$ along the field line from an arbitrary base level. Considering the protonosphere where chemical production and loss processes are ignorable and denoting the ion-electron density by $\mathrm{n}$, the sum of the ion and electron temperatures by $\mathrm{T}$, and the flow velocity in the direction of the magnetic field by $v$, the one dimensional equations of continuity, momentum and energy for adiabatic flow are:

$$
\begin{gathered}
n_{t}+v n_{s}+n v_{s}=\frac{n v}{B} B_{s} \\
n v_{t}+n v v_{s}+\frac{k T}{M} n_{s}+\frac{k}{M} n T_{s}=-n g \\
n\left(T_{t}+v T_{s}\right)+(1-\gamma) T\left(n_{t}+v n_{s}\right)=0 .
\end{gathered}
$$


In these equations subscripts denote partial differentiation, B denotes the magnetic field, $\mathrm{k}$ is Boltzman's constant, $\mathrm{M}$ is the proton mass, $\mathrm{g}$ is the magnitude of the gravitational acceleration component in the direction of the magnetic field and $\gamma$ is the ratio of specific heats. When isothermal flow is considered only equations (1) and (2) are applicable with $T_{s}=0$.

The set of hyperbolic equations (1) through (3) are best solved numerically by the method of characteristics. Since this is a technique that has been thoroughly described in the literature (see for example, Courant and Friedricks, 1948) the solution scheme will not be described in detail. For the particular physical model at hand the numerical solution follows the development employed by Grebowsky (1968) and Grebowsky and Smith (1971).

In order to obtain a complete solution of equations (1) through (3) at all times and at all points along a field line, proper boundary conditions must be specified. The natural choice for the boundary conditions is a specification of $\mathrm{n}, \mathrm{v}$ and $\mathrm{T}$ at all points along the field line at time $\mathrm{t}=0$ and at all times at the base of the field line (i.e., at $s=0$ ). Symmetry of the plasma flow along a trough field line about the equatorial plane is assumed so that the condition $v=0$ at the equator completes the set of required boundary conditions. Due to the assumed symmetry of the flow about the magnetic equator the solution will be developed explicitly on only one side of the equator.

The subsonic region on the equator side (i.e., downstream) of the shock is coupled directly to the upward directed supersonic flow on the earth side via the 
shock jump conditions which are derived by requiring the conservation of plasma number flux along with the conservation of momentum and energy fluxes through the shock discontinuity. Defining the acoustic speed by $\mathrm{c}^{2}=\gamma \mathrm{kT} / \mathrm{M}$, the relationship between the dependent variables on the equator side of the shock (i.e., in the subsonic region) denoted by the subscript 1 and those on the earth side of the shock, denoted by the subscript 0 , as obtained from the conservation relations are:

$$
\begin{gathered}
\frac{\mathrm{n}_{0}}{\mathrm{n}_{1}}=\frac{(\gamma-1) \mathrm{v}_{0}^{\prime 2}+2 \mathrm{c}_{0}^{2}}{(\gamma+1) \mathrm{v}_{0}^{\prime 2}} \\
\frac{\mathrm{v}_{1}^{\prime}}{\mathrm{v}_{0}^{\prime}}=\frac{\mathrm{n}_{0}}{\mathrm{n}_{1}} \\
\mathrm{c}_{1}^{2}=\left(\frac{\mathrm{n}_{0}}{\mathrm{n}_{1}}\right)\left[\mathrm{c}_{0}^{2}-\gamma \mathrm{v}_{0}^{\prime}\left(\mathrm{v}_{1}^{\prime}-\mathrm{v}_{0}^{\prime}\right)\right]:
\end{gathered}
$$

The primes denote velocities in the rest frame of the moving shock. That is, $v^{\prime}=v-V_{s}$ where $V_{s}$ is the shock velocity and $v$ is the observed velocity in the frame of reference fixed with respect to the plane of the field line. Using the characteristic equations resulting from the hydrodynamic relations (1) through (3) and using relations (4) through (5) to couple the supersonic and subsonic regions, the time evaluation of the plasma flow along trough field lines can be calculated. 
Fixed L Coordinate

Setting the $\mathrm{L}$ coordinate at a value of 6 , the evaluation of the plasma flow is computed from an initial state corresponding to the upward directed supersonic steady state flow solution of the hydrodynamic equations from the base of the field line to the equator where the shock discontinuity originally forms. The nature of similar flow states along field lines characterized by different $\mathrm{L}$ coordinates can be inferred from these calculations and the previous computations of Banks et al. (1971).

The base level altitude is selected as 3000 kilometers which at trough latitudes is in the protonosphere (Brinton et al., 1971). Typical values chosen for the plasma density and temperature at this altitude where the upward plasma flow is assumed to be slightly supersonic are:

$$
\begin{aligned}
& \mathrm{n}=300 \text { ions-electrons } / \mathrm{cc} \\
& \mathrm{T}=8000^{\circ} \mathrm{K} \\
& \mathrm{v}=11.0 \mathrm{~km} / \mathrm{sec}
\end{aligned}
$$

The temperature $8000^{\circ} \mathrm{K}$ corresponds to a sound speed c of $10.49 \mathrm{~km} / \mathrm{sec}$ for adiabatic flow $(y=5 / 3)$ and to $8.13 \mathrm{~km} / \mathrm{sec}$ for isothermal flow $(\gamma=1)$. These values will be used as the assumed time invariant boundary values for all the consequent model calculations.

\section{Model Calculations}

Under the assumption that thermal conduction is sufficient on either side of the shock to produce an isothermal state, the flow evolves as shown in Figure 2. 
The initial upward directed supersonic flow corresponding to steady state conditions is an upward accelerated flow. As seen in the figure, initially the shock is a discontinuity at the equator with vanishing plasma flow and enhanced temperature and density at the equator.

As the shock moves away from the equator the supersonic flow on the earth side remains unchanged whereas the downstream subsonic flow varies with time. The shock velocity decelerates from approximately $12 \mathrm{~km} / \mathrm{sec}$ when the shock is initially at the equator to values less than $0.1 \mathrm{~km} / \mathrm{sec}$ when the shock reaches altitudes near and below 10,000 kilometers. This is in agreement with the study of Banks et al., (1971) which found that the shock takes the order of a day or two to travel from the equator to the base of the protonosphere. If this model were applicable for longer periods of time, the shock would disappear and the flow along the entire field line would become subsonic since the decelerating shock must become weaker as the flow upstream of the shock decelerates with decreasing altitude towards subsonic values.

The temperature is enhanced up to a factor of ten fold through the shock transition and hence would result in a large scale height for the density variation at high altitudes. However, the plasma state departs significantly from diffusive equilibrium due to the relatively large magnitude of the subsonic flow velocity downstream of the shock. This is particularly evident since the computed variation of the subsonic velocity yields an increase of the plasma density with increasing altitude downstream of the shock. 
The temperature on the equator side of the shock drops from an initial peak of $88,000^{\circ} \mathrm{K}$ to values near $24,000^{\circ} \mathrm{K}$ under isothermal conditions as the shock moves earthward. If one arbitrarily assumes that the ion and electron temperatures are comparable to one another, the computed temperatures correspond to individual species energies of the order of a few ev. Energies of this order of magnitude have been observed for the thermal ions outside of the plasmasphere (Serbu and Maier, 1970).

If on the other hand the flow was adiabatic along the field line, the flow would evolve as shown in Figure 3 from the previously described initial conditions. It is not likely that the actual flow state is accurately described by the adiabatic model since the adiabatic steady state solution is characterized by a decrease of the temperature with increasing altitude to values less than $1000^{\circ} \mathrm{K}$ - magnitudes which are much lower than experimentally measured. However, the adiabatic model will provide limits for the actual flow and by comparison with the isothermal results will contribute to an understanding of the possible flow states.

Again, as for isothermal flow, the shock decelerates as it moves earthward and a period of several days is required for the shock to travel from the equator to the base of the protonosphere. As the shock moves earthward the density everywhere on the equator side increases with time due to the upward plasma transport. However, the rate of increase of the ion density at the equator is slower than the increase near the shock so that a narrowing depression in the 
density occurs centered on the equator. Associated with the density depression is a peak in the temperature due to the deposition of energy by the equatorially converging plasma streams. The adiabatic flow is thus characterized by upward directed temperature gradients in the subsonic flow region.

The previous calculations for a fixed $L$ coordinate indicate the general behavior expected when upward directed supersonic streams form plasma shocks on closed magnetic field lines. Both the adiabatic and isothermal models predict significant departures from diffusive equilibrium in the expanding subsonic region equatorward of the shock. Also both models predict that the shock can take several days to travel from the equator to the base of the protonosphere. In order to determine whether this behavior is always characteristic of the trough flow, the assumption of a time invariant $L$ coordinate must be relaxed in order to account for the effects of the more complex convection patterns known to exist outside of the plasmasphere. The next section will explore the effects of a varying $L$ coordinate.

\section{Cross L Drift}

The general magnetospheric convection pattern for the thermal plasma outside of the plasmasphere is characterized by cross $L$ drifts. These cross $L$ drifts can be explored using the hydrodynamic one dimensional time dependent equations to describe the plasma motion along the field line as the field line and the plasma frozen to it contract (expand) with a decrease (increase) in the $L$ coordinate. 
The procedures for including the temporal variation of the $\mathrm{L}$ coordinate within the framework of the one dimensional hydrodynamic equations (1) through (3) consists of approximating the continuous change of the $L$ coordinate by a discrete variation in which the $\mathrm{L}$ coordinate is impulsively changed at small time intervals. Assuming that the $L$ coordinate is specified as a function of time, the solution scheme for the flow development consists of the following steps:

1. The plasma state is specified at equidistant points along the magnetic field line characterized by the coordinate $L(t)$ at time $t$. With the $L$ coordinate fixed at the value $L(t)$ the hydrodynamic and shock relations are solved to determine the solution at each computing point at time $t+\Delta t$

2. The $L$ coordinate is changed to $L(t+\Delta t)$ and the dependent variables at equidistant points along the $L(t+\Delta t)$ line are obtained by a one to one mapping of the equidistant computing points along the $L(t)$ line. In this transformation the total ion tube content is conserved and the density will vary inversely to the incremental change in volume associated with each transformed point. For an isothermal state the temperature at a point will remain unchanged. However, for adiabatic flow the temperature is transformed according to the relation $\operatorname{Tn}^{-\gamma+1}=$ constant. The velocity at each point remains unchanged in the transformation except at the downstream side of the shock where in order to conserve the total 
ion content and to preserve the quasistatic nature of the shock process the downstream velocity must be computed from the shock relations (4) through (6).

3. Replacing $t$ by $t+\Delta t$ the solution can then be obtained at a later time following the above steps.

\section{Field Line Contraction}

In order to determine what effect a time dependent $\mathrm{L}$ coordinate has on the field aligned flow, an extreme situation will be treated in which the $L$ variation is taken as that characteristic of the inward magnetosphere drift expected at midnight for a storm enhanced convective flow state. A simple model often used (e.g., Kavanaugh et al., 1968; Chen and Wolf, 1971) to approximate the convective flow from the magnetosphere tail describes the $\vec{E} \times \vec{B}$ drift of the ambient plasma in the equatorial plane as due to a constant dawn to dusk directed electric field. The magnitude of this field is typically of the order of a few tenths of a millivolt/meter. Using this model and assuming a dipole field line is characterized initially by the $L$ coordinate $L=11$ (i.e., a field line that extends into the magnetospheric tail) the variation of $L$ along a convection streamline at midnight for $E=0.5 \mathrm{mv} / \mathrm{m}$ corresponding to an enhanced flow state is:

$$
L=\frac{11}{\sqrt{2.13 t+1}}
$$

This functional variation which ignores the effects of corotation will be employed to determine the gross effects of the cross $L$ drift on the development of the high speed flow along trough field lines. 
Again, as previously, the temporal development is computed for both adiabatic and isothermal flow states beginning with the steady state solution along the $\mathrm{L}=11$ field line at time $\mathrm{t}=0$. The isothermal flow development under the cross $L$ drift of equation 7 is shown in Figure 4.

As the plasma convects inward to smaller L coordinates, the flow in the supersonic region varies with time as the plasma state continually relaxes towards the steady state configurations associated with each contracted field line state. Only the velocity variations in the supersonic region are plotted in detail in Figure 4 - the corresponding density variations in the supersonic region were small compared to the initial profile and were omitted for clarity. The temperature on the earth side of the shock was fixed at $8000^{\circ} \mathrm{K}$.

The general features of each of the computed field line states are similar to those derived previously with the I coordinate held fixed. The density in the subsonic region varies only slightly with altitude along the field lines except in the immediate neighborhood of the shock where large gradients in the flow speed can develop. The temperature on the equator side of the shock decreases with time while the density increases.

Since the cross $\mathrm{L}$ drift model employed approximates the convection inward at midnight outside of the plasmasphere, the temporal variations computed at the equator correspond to altitude variations. The computed temperature increases with altitude attaining values of the order of $90,000^{\circ} \mathrm{K}$. Also due to the upward transport of plasma the density decreases with altitude at the equator. This density 
decrease differs significantly from the $1 / L^{4}$ variation which would exist if the cross L drift was not accompanied by a flow of plasma along the field lines.

The shock, as in previous calculations, decelerates in its motion towards the earth. The sensitivity of the shock velocity to variations in the flow resulting from the contraction is apparent in the oscillatory variation of the shock velocity. Originating at the equator on the $\mathrm{L}=11$ field line, the shock reaches the base of the field line in a period of a few hours at which time it has drifted to an $\mathrm{L}$ coordinate less than 4. The exact time and location of this transit is of course dependent upon the functional form of the cross $\mathrm{L}$ drift.

For the same variation of the $\mathrm{L}$ coordinate with time an adiabatic flow state will evolve as shown in Figure 5. The contraction of the flux tube induces compression and rarefraction waves which interact with the shock to produce fluctuations in the magnitude of the shock velocity. After an initial downward acceleration period near the equator the shock tends to decelerate as it moves into the denser plasma near the earth. The shock reaches the base altitude in three hours when it has drifted near the $\mathrm{L}=4$ field line.

As in the fixed $L$ study the adiabatic flow configurations exhibit more complex structure than the isothermal development. For $y=5 / 3$ the density on the equator side of the shock decreases with altitude along the field lines resulting in an extensive trough in the density at the equator. The temperature on the other hand peaks at the equator as energy is transferred to the equator via the plasma motion along the field line and as the plasma is adiabatically heated by the 
compression of the flux tube. Assuming the field line drifts within a fixed magnetic meridian, the computed temporal development corresponds to an equatorial temperature which increases rapidly with decreasing distance from the earth. It varies from $15,000^{\circ} \mathrm{K}$ (of the order of $1 \mathrm{ev}$ ) at $\mathrm{L}=11$ to values near $150,000^{\circ} \mathrm{K}$ (of the order of $10 \mathrm{ev}$ ) at $\mathrm{L}=4$. Hence adiabatic flow produces an extremely hot plasma state just outside of the plasmasphere.

Another interesting feature of the adiabatic development is the production of back pressures on the equator side of the shock which drive the subsonic flow away from the equator during the initial phase of the shock motion. Eventually however the flow throughout the subsonic region is directed upward along the field line. Hence in contrast to the isothermal model the subsonic flow can be directed in either direction along the field line with the downward flows prevailing at the lower $\mathrm{L}$ coordinates.

Expanding Flux Tube

Only a compression of a trough flux tube via convection to smaller L coordinates was explicitly treated previously. However, the flow model under consideration can be extended to include cross $L$ drifts in which the plasma convects in the direction of increasing $L$ coordinates. Such drifts occur in the magnetosphere since the convective flow from the magnetosphere tail completes its cycle by traversing the magnetopause.

The effects of a drift to higher L coordinates were explored by considering the flow evolution when the $\mathrm{L}$ coordinate varied according to the reverse of 
equation (7). The initial states for the expansion were chosen to be the solutions computed in the previous section at $\mathrm{L}=4.8$. The results are depicted in Figure 6. Only the shock velocity and variations at the equator will be considered explicitly since the other variations are analogous to those computed previously. The initially earthward moving shocks (negative velocities) decelerate until the reduction of pressure downstream by the expansion process results in a reversal of the shock motion at $L$ coordinates slightly greater than 5 . The shocks then accelerate upwards as they drift to higher L coordinates. Thus following a typical drift path of a trough field line in the magnetosphere, along which compression and expansion occur, the cross $\mathbf{L}$ drift from the magnetosphere tail towards lower $\mathrm{L}$ coordinates will be characterized by downward moving shocks; whereas, the convection in the direction of increasing $L$ coordinates can be characterized by upward moving shocks as the region of supersonic flow expands along the field direction towards the steady state polar wind configuration.

During the expansion under isothermal conditions the equatorial temperature initially decreased with increasing $L$ but as the shock moved towards the equator the temperature slowly increased. For adiabaticity, on the other hand, the equatorial temperature always decreased with time since the adiabatic expansion of the flux tube cooled the plasma faster than it could be heated by energy transport along the field line. In both cases the equatorial density decreased with increasing distance from the earth. In fact the equatorial density closely follows a $1 / \mathrm{L}^{4}$ relation indicating that the flux tube expansion affects the density more than the transport along the field line. 


\section{Conclusions}

The evolution of the plasma flow along trough field lines has been computed for the case of a fixed $\mathrm{L}$ coordinate and for cross $\mathrm{L}$ drifts in which the $\mathrm{L}$ coordinate decreases and increases with time. These calculations yield the general flow behavior expected when the supersonic trough wind is transformed via a shock discontinuity into a subsonic flow. The effect of the shock is to produce a region of hot plasma centered about the equator in which the plasma can depart significantly from a static diffusive equilibrium state.

Regions of supersonic flow are always expected along the closed field lines with high L coordinates outside of the plasmosphere since the time required for the shock to travel from its initial position at the equator to below the base of the protonosphere, where the supersonic flow does not exist, is typically much longer than the lifetime of a convecting closed field line. The field lines with smaller L coordinates near the plasmapause on the other hand may at times be characterized by only subsonic field aligned flows because the shock quickly travels the length of these lines of force. Even these subsonic flows however will eventually become supersonic as the plasma drifts in the direction of increasing $L$ with an attendent upward acceleration which produces supersonic flow and an upward moving shock. The expending supersonic region must approach the polar wind configuration as the $\mathrm{L}$ coordinate increases toward polar values.

Thus the dynamics of the ambient plasma along trough field lines is seen to be very complex with the plasma state along any given field line a function of 
the past history of its drift motion. Extensive measurements of the ambient plasma distribution along the trough field lines are now required to set more realistic and flexible boundary conditions and to test these models. 


\section{BIBLIOGRA PHY}

Banks, P. M. and T. E. Holzer, "High Latitude Plasma Transport: The Polar Wind," J. Geophys. Res., 74, 6317, 1969a.

Banks, P. M. and T. E. Holzer, "Features of Plasma Transport in the Upper Atmosphere," J. Geophys. Res., 74, 6304, $1969 \mathrm{~b}$.

Banks, P. M., A. F. Nagy, W. I. Axford, 'Dynamical Behavior of Thermal Protons in the Mid-Latitude Ionosphere and Magnetosphere," Planet. Space. Sci., 19, 1053, 1971 .

Brinton, H. C., J. M. Grebowsky, H. G. Mayr, "Altitude Variation of Ion Composition in the Midlatitude Trough Region: Evidence for Upward Plasma Flow," J. Geophys. Res., 76, 3738, 1971.

Chen, A. J., and R. A. Wolf, "Effects on the Plasmasphere of a Time-Varying Convection Electric Field," Planet. Space Sci., 19, , , 1971.

Courant R. and K. O. Friedricks, "Supersonic Flow and Shock Waves," Interscience Publishers, 1948.

Grebowsky, J. M., "Model Studies of Low Energy Plasma in the Outer Magnetosphere," Ph.D. Thesis, The Pennsylvania State University, June 1968.

Grebowsky, J. M. and R. K. Smith, "Solution Scheme for Time Dependent Hydrodynamic Plasma Flow Along a Magnetic Field Line," NASA, Goddard Space Flight Center Document X-621-71-173, April, 1971.

Holzer, T. E., J. A. Fedder, P. M. Banks, "A Comparison of Kinetic and Hydrodynamic Models of an Expanding Ion- Exosphere," J. Geophys. Res., 76, 2453, 1971. 
Kavanagh, L. D., J. W. Freeman, A. J. Chen, "Plasma Flow in the Magnetosphere," J. Geophys. Res., $\underline{73}, 5511,1968$.

Koons, H. C. and M. Schulz, "Thermalization of Colliding Ion Streams Beyond The Plasmapause," (abstract), Eos Trans. AGU, 51, 804, 1970.

Mayr, H. G., J. M. Grebowsky and H. A. Taylor, Jr., "Study of the Thermal Plasma on Closed Field Lines Outside the Plasmasphere," Planet. Space Science, $18,1123,1970$.

Schulz, M. and H. C. Koons, "Thermalization of Colliding Ion Streams Beyond the Plasmapause," J. Geophys. Res., 77, 248, 1972.

Serbu, G. P. and E. J. R. Maier, "Observations from OGO 15 of the Thermal Ion Density and Temperature within the Magnetosphere," J. Geophys. Res., $\underline{75}, 6102,1970$. 

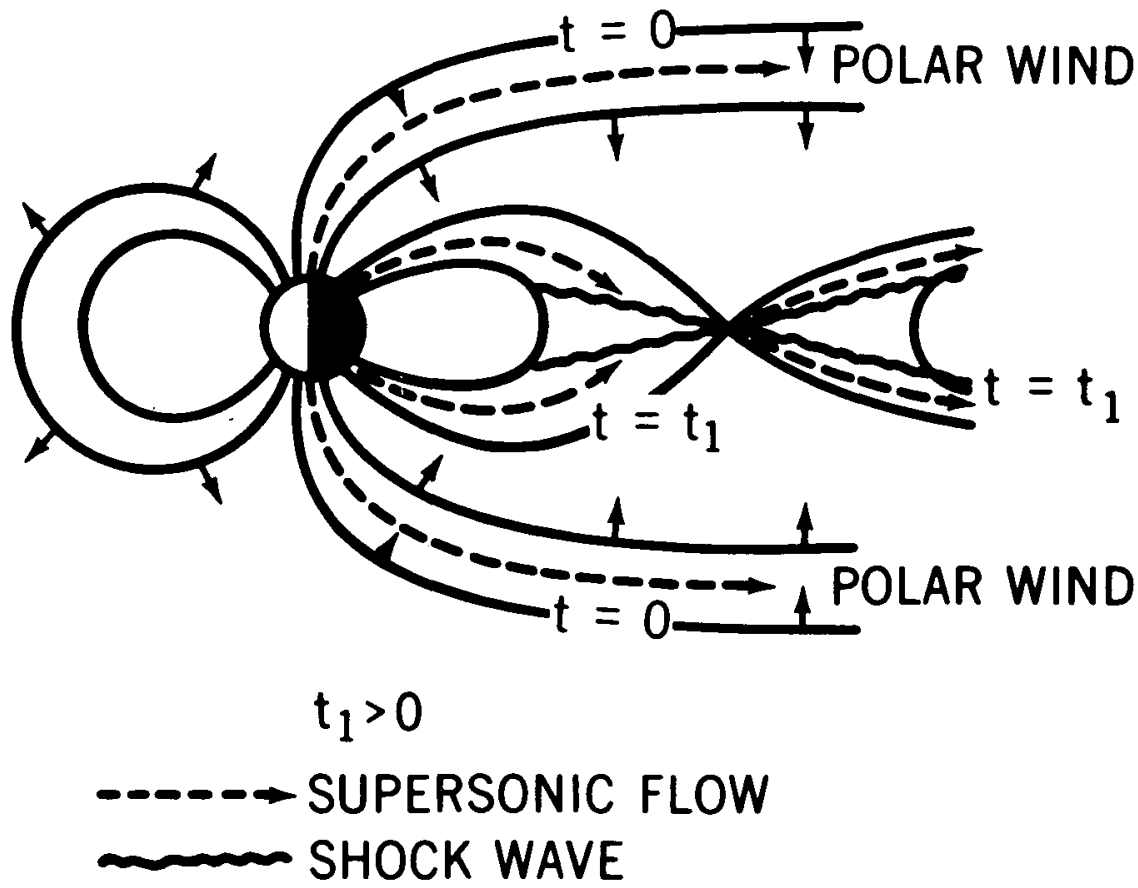

Figure 1. Polar wind convects onto closed field lines at night resulting in shocks which propagate towards the earth as the plasma drifts around the earth to the dayside magnetopause where the polar wind evolves. 

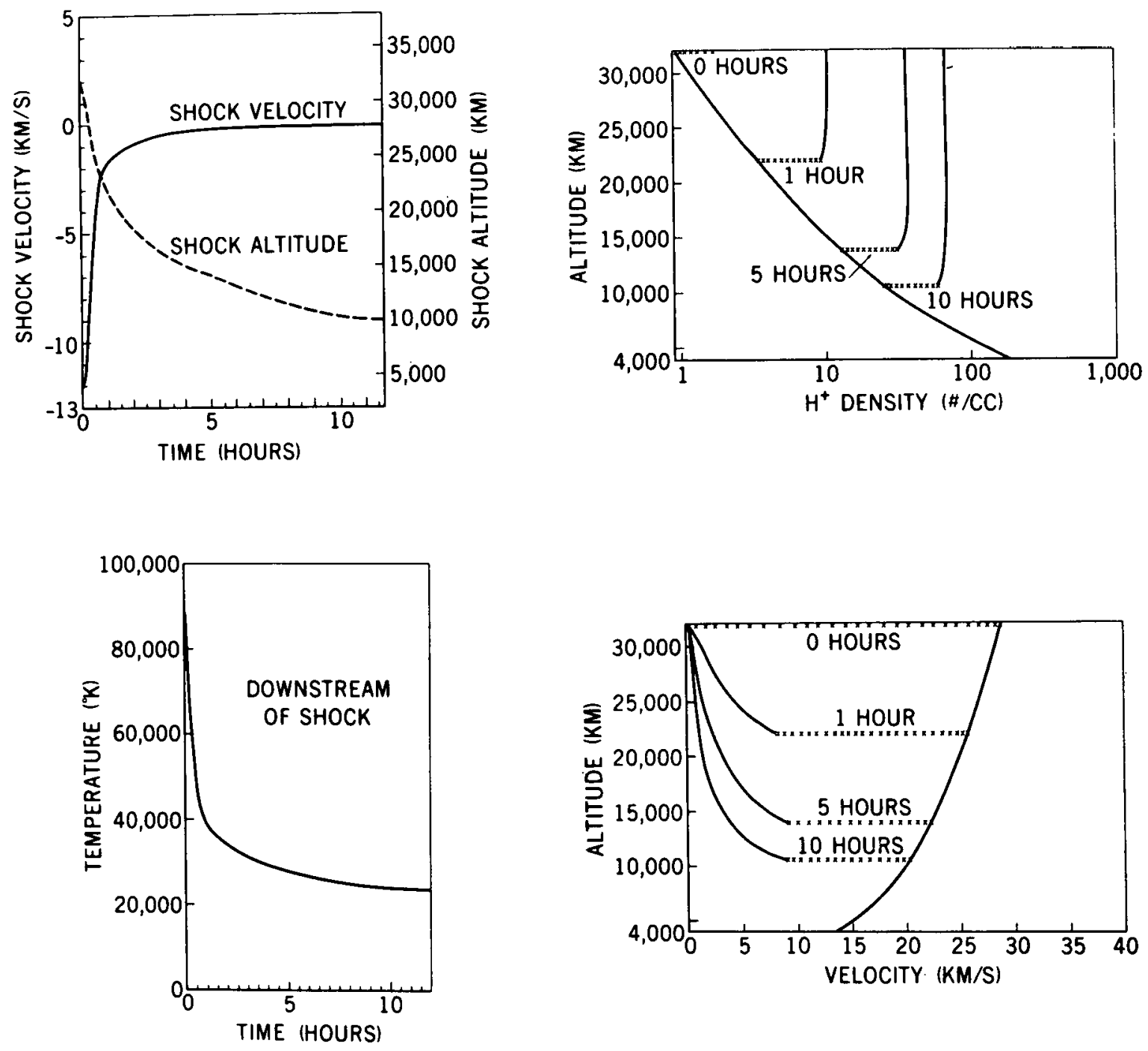

Figure 2. The temporal development of the plasma flow along an $L=6$ magnetic field line is plotted for isothermal conditions. The shock is at the equator initially and the supersonic flow on the earth side of the shock corresponds to the steady state solution of the hydrodynamic equations. 


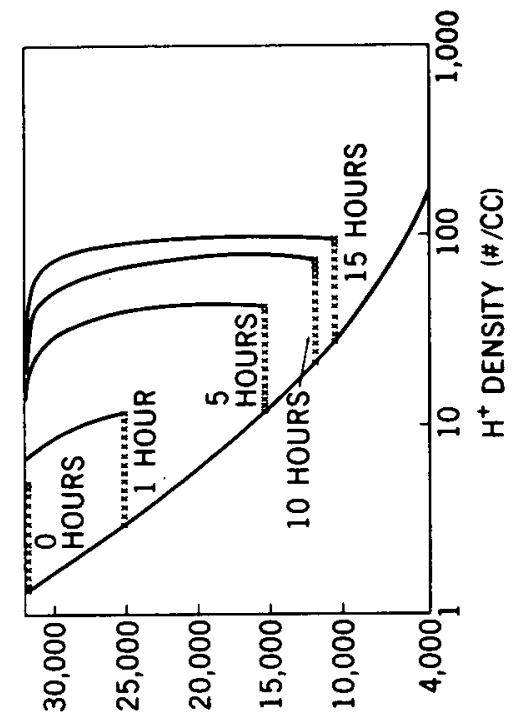

(WX) $30 \cap 1117 \forall$

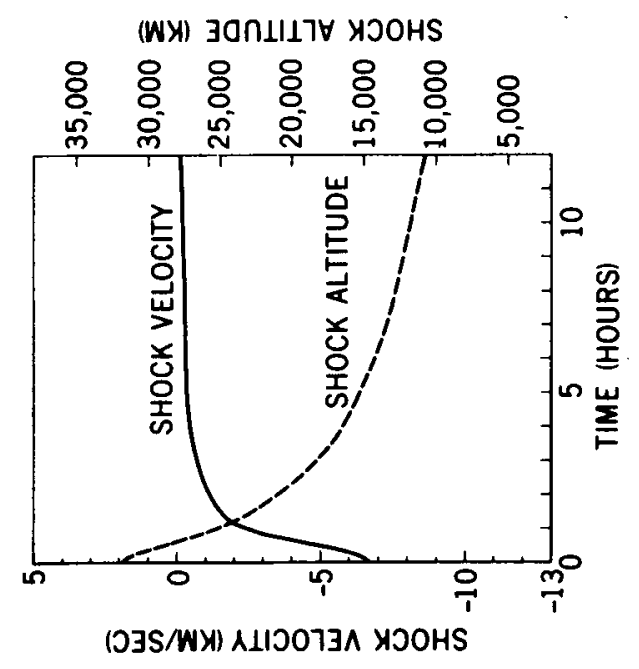

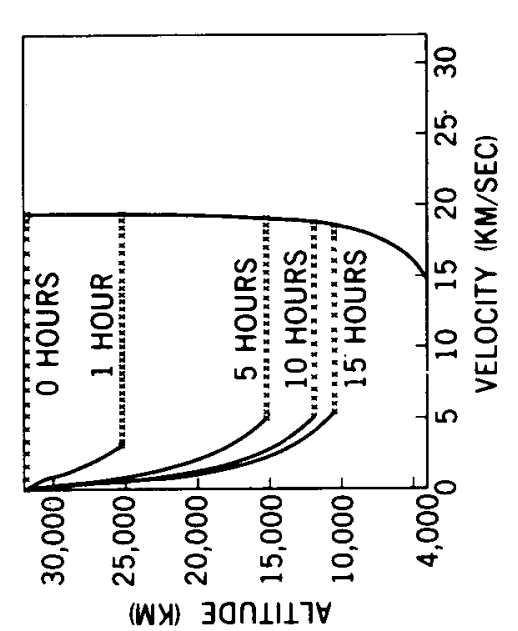

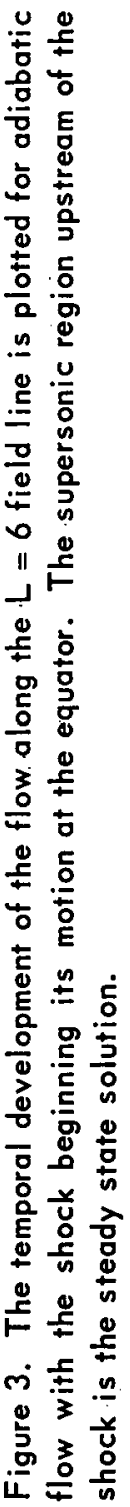

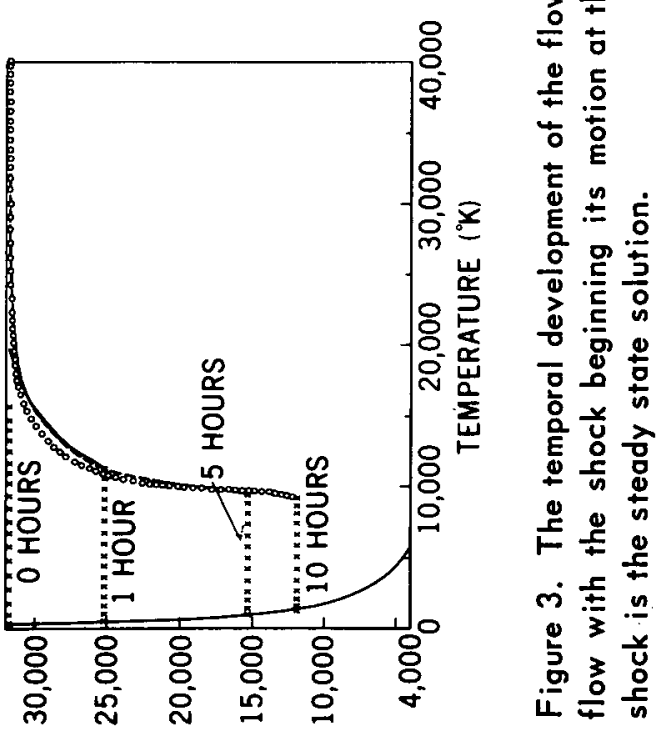

(Wy) $30 \cap 1117$ 

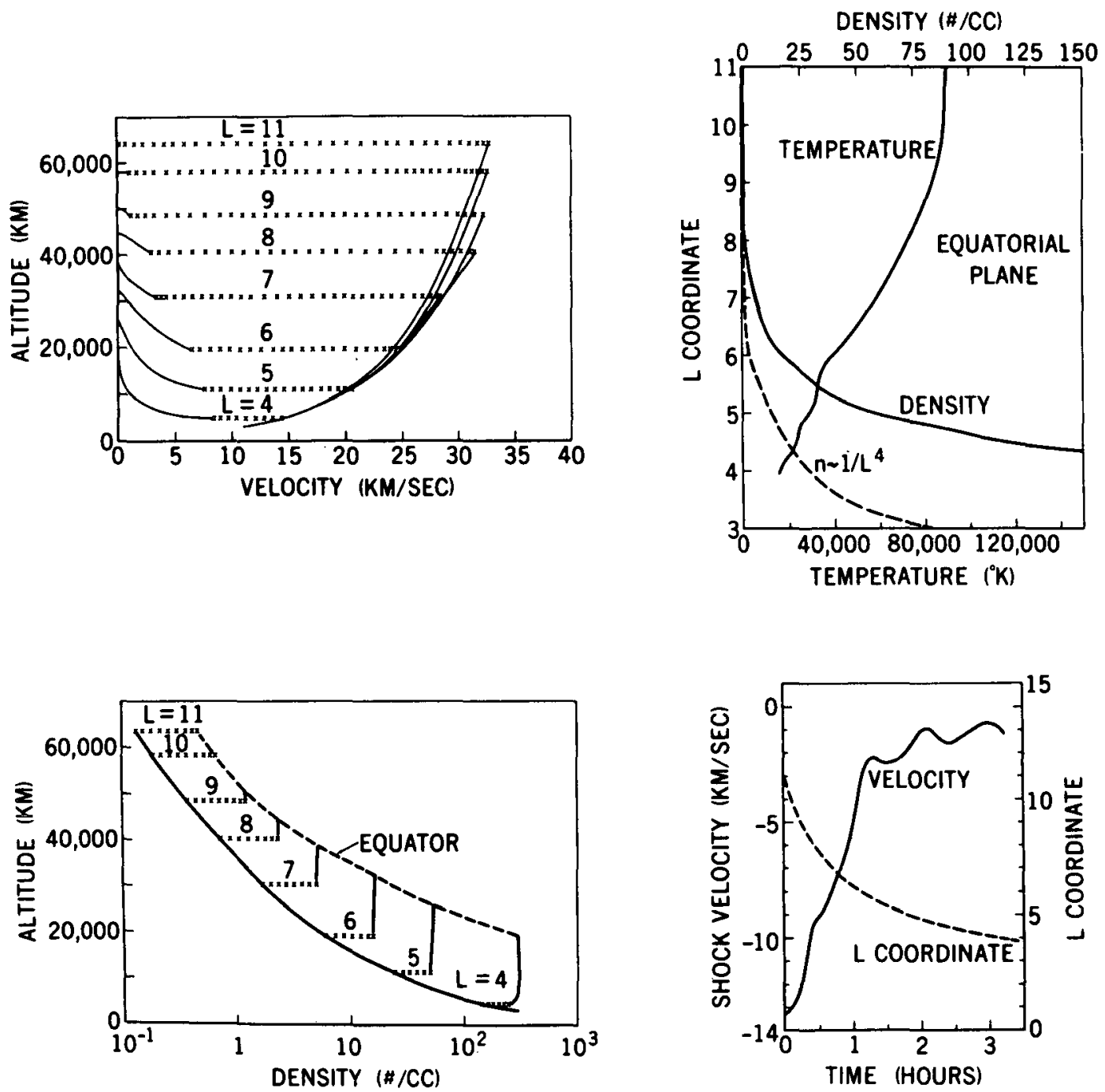

Figure 4. For isothermal flow the field akigned distribution evolves as shown when the field line and its frozen in plasma drifts in the direction of increasing $L$ occording to equation 7 . The initial distribution along the $L=11$ line is the steady state supersonic flow. 

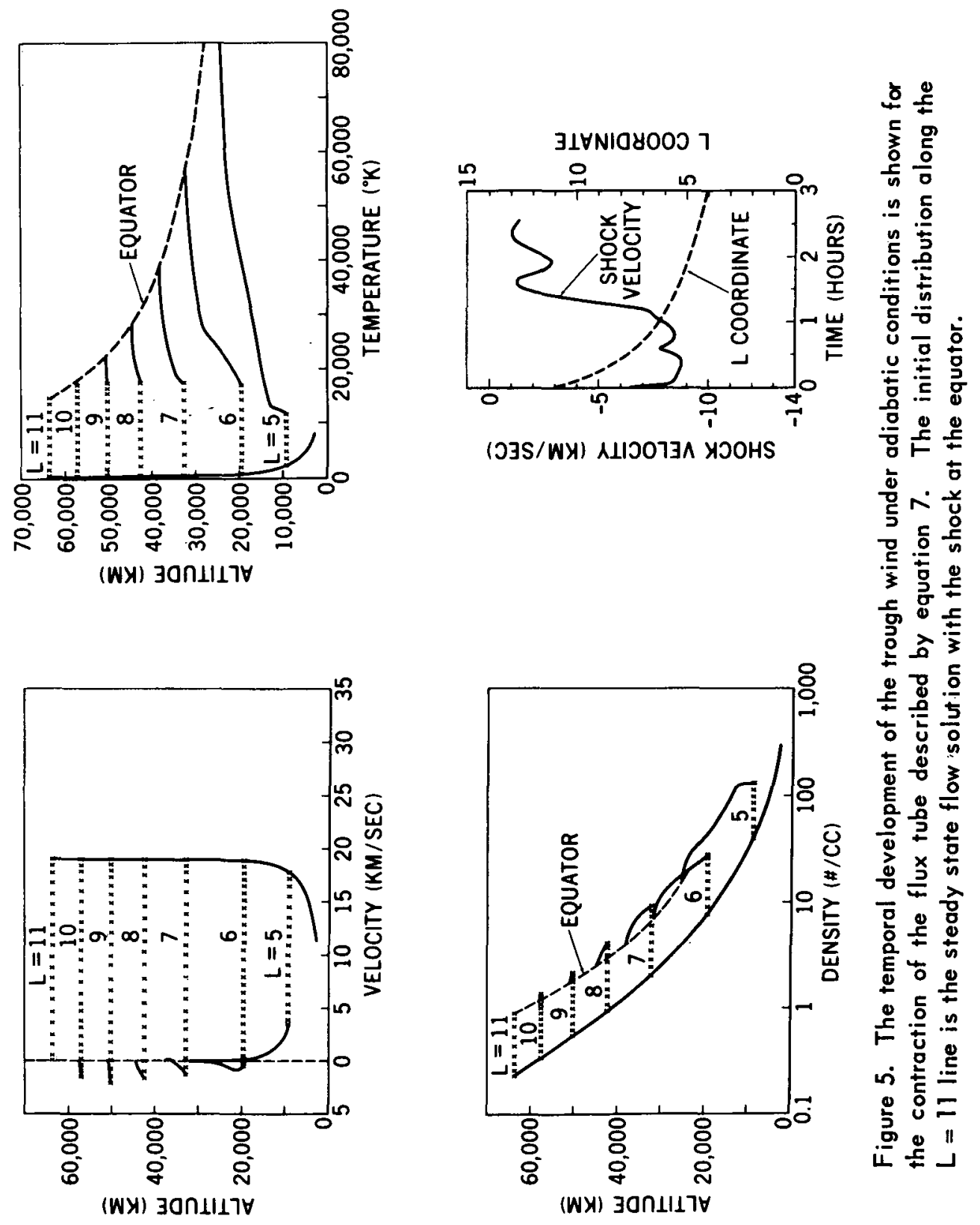

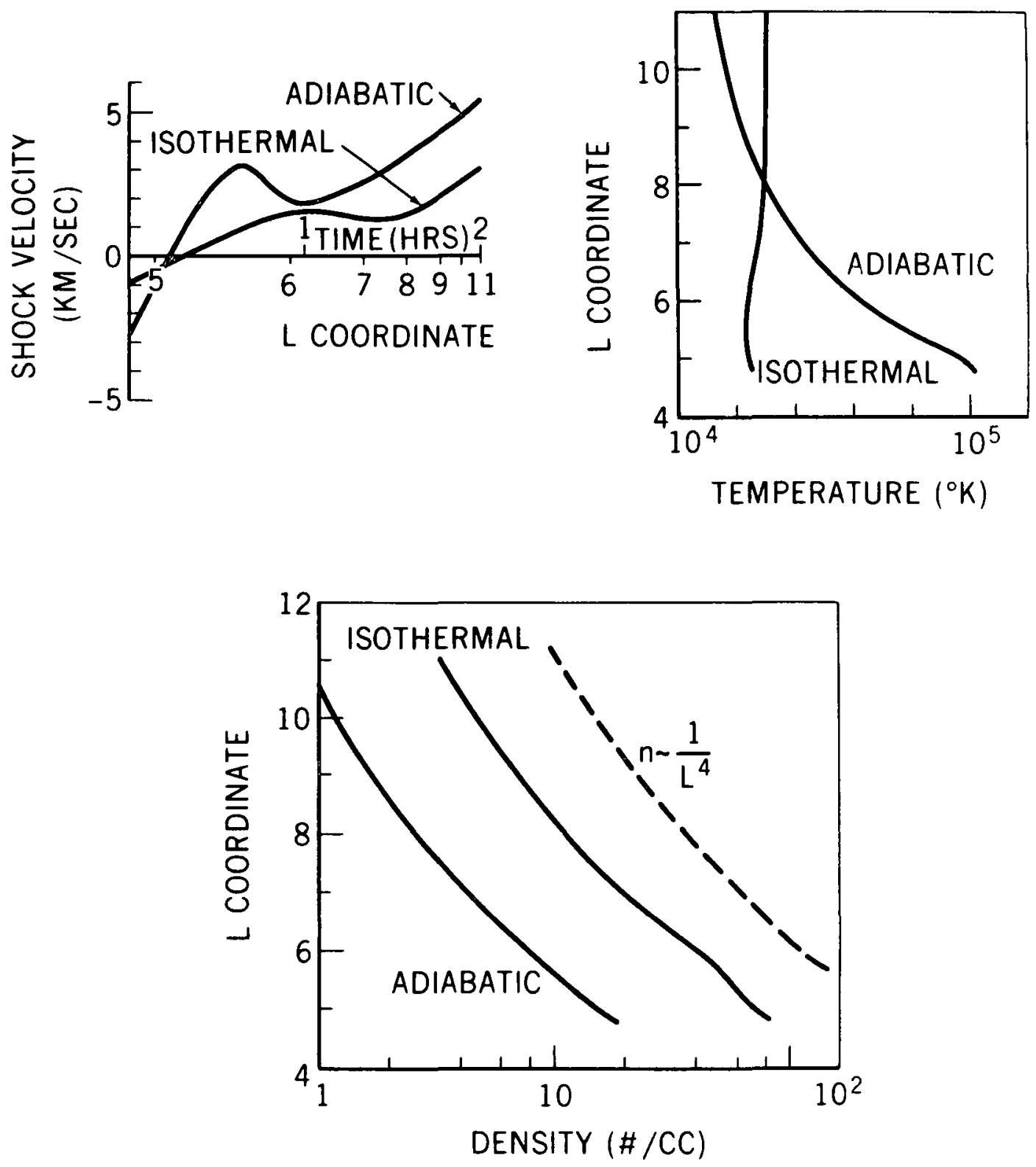

Figure 6. The variation of the shock velocity and the equatorial plasma state for both adiabatic and isothermal flow is described for a flux tube which expands according to the inverse of equation 7. The initial states are the solutions produced for the contraction of the flux tube to smaller $L$ coordinates. 